\title{
Treatment of bone and soft tissue tumors of the limbs with conformal radiotherapy and intensity-modulated radiotherapy (IMRT)
}

\author{
TRATAMENTO DE TUMORES ÓSSEOS E DE PARTES MOLES DE MEMBROS COM RADIOTERAPIA \\ EXTERNA CONFORMADA E COM INTENSIDADE MODULADA (IMRT)
}

\author{
Authorship: Brazilian Society of Radiotherapy (SBR) \\ Participants: Marcus Simões Castilho ${ }^{1}$, Robson Ferrigno ${ }^{1}$, Helena Baraldi ${ }^{1}$, \\ Paulo Eduardo Ribeiro dos Santos Novaes ${ }^{1}$
}

${ }^{1}$ Sociedade Brasileira de Radioterapia (SBR)

The Guidelines Project, an initiative of the Brazilian Medical Association, aims to combine information from the medical field in order to standardize procedures to assist the reasoning and decision-making of doctors.

The information provided through this project must be assessed and criticized by the physician responsible for the conduct that will be adopted, depending on the conditions and the clinical status of each patient.

\section{Grades of RECOMMENDATION AND LEVELS OF EVIDENCE}

- A: Experimental or observational studies of higher consistency.

- B: Experimental or observational studies of lower consistency.

- C: Cases reports (non-controlled studies).

- D: Opinion without critical evaluation, based on consensus, physiological studies or animal models.

\section{DESCRIPTION OF EVIDENCE COLLECTION METHOD}

Through the elaboration of three relevant clinical questions related to the proposed theme, we sought to present the main evidences regarding safety, toxicity and effectiveness of the presented radiotherapy techniques. The study population consisted of male and female patients of all ages with bone and soft tissue tumors in the upper and lower limbs, regardless of histological type, staging, treatment context (neoadjuvant, radical or adjuvant) or the presence of comorbidities. For this, a systematic review of the literature was carried out in primary scientific databases (MEDLINE - PubMed; Embase - Elsevier; LILACS - BIREME; Cochrane Library - Record of Controlled Trials). All articles available through May 31, 2015 were considered. The search terms used in the research were: (conformal radiotherapies [MeSH Terms]) OR radiotherapies, conformal [MeSH Terms]) OR conformal radiotherapy [MeSH Terms]) OR radiotherapy, intensity-modulated [MeSH Terms]) OR conformal radiotherapy) OR conformal radiotherapies) OR conventional radiotherapy) OR IMRT) OR 3D conformal radiotherapy) OR VMAT)) AND (sarcomas [MeSH Terms]) OR sarcoma, soft tissue [MeSH
Terms]) OR sarcomas, soft tissue[MeSH Terms]) OR soft tissue sarcoma [MeSH Terms]) OR sarcoma) OR sarcomas).

The articles were selected based on critical evaluation using the instruments (scores) proposed by Jadad and Oxford. The references with greater degree of evidence were used. The recommendations were elaborated after discussion with the elaboration group composed by four members of the Brazilian Society of Radiotherapy.

\section{Objective}

To evaluate the most appropriate technique of radiotherapy for the treatment of patients with bone and soft tissue tumors of the limbs.

\section{INTRODUCTION}

The therapeutic strategy of bone and soft tissue tumors of the limbs should be performed through multidisciplinary decision to better associate surgery, radiotherapy and chemotherapy. The decision on the best combination and sequence is based on factors such as tumor type and histological grade, clinical staging, primary tumor location and volume, proposed type of surgery, and general patient conditions. External radiotherapy can be used preoperatively (neoadjuvant) or postoperative (adjuvant) and aims to ensure local control of the primary tumor before or after surgery.

Although there are no viscera or vital organs in the limbs, the joints and soft tissues are susceptible to severe complications of radiotherapy, such as lymphedema, joint stiffness, soft tissue fibrosis, and necrosis of bones and soft tissues. If an irradiated bone fractures, there is no consolidation of this fracture due to changes in the mi- 
crovasculature caused by irradiation. These sequelae may lead to limitation of limb functions, such as articulation and movement, and consequent impairment of quality of life. In some situations, sequelae may leave the patients disabled and, depending on their profession, drive them out of the job market.

Therefore, the radiation dose should be directed as concentrated as possible to the area at risk of local recurrence after surgery or in the primary tumor prior to surgery, also minimizing or avoiding the therapy in areas at risk that do not need to be treated, such as those with soft tissues, bones, tendons, vessels, nerves, muscles and joints.

In recent years, the technical development of radiotherapy has allowed the planning of the radiation deposit to be made using imaging tests, which is not performed with conventional radiotherapy, but with conforming techniques and IMRT.

Conventional radiotherapy is performed from simple radiographs of the affected limb, on which the area to be irradiated is drawn in two dimensions. Dose calculation is done manually taking into account the size of the field and the thickness of the limb. This technique can not estimate the amount of dose released in areas that do not require treatment. Because of these characteristics, conventional radiotherapy has been abandoned for several years in developed countries and is not recommended by the Brazilian Society of Radiotherapy for the treatment of limb tumors. ${ }^{1}$ (D)

Conformal radiotherapy was developed so that the planning would be performed with imaging tests such as computed tomography, and the calculation of the dose with computerized systems that evidence the dose distribution in three dimensions. With this, it is possible to estimate if the dose distribution is adequate and safe. The planning system allows various combinations of radiation input fields to be modified in their incidence and size, in order to focus the radiation only where it is needed. Thus, radiotherapy has become potentially safer and more effective for patients with bone and soft tissue tumors of the limbs.

IMRT radiotherapy was developed from conformal radiotherapy with the addition of a planning system that can place radiation filters in the front of the beam so that the homogeneity of dose is greater, avoiding excessive doses and bypassing risk structures more efficiently.

Based on these premises, this guideline aims to show the benefits of the most modern techniques of radiotherapy in the management of patients with bone and soft tissue tumors of the limbs, with the support of published dosimetric and clinical evidence.
To that end, three questions were elaborated to answer the main points.

\section{IS THERE A DOSIMETRIC SUPERIORITY IN THE IRRADIATION OF SOFT TISSUE AREAS WITH CONFORMAL RADIOTHERAPY OR INTENSITY- -MODULATED RADIATION BEAM (IMRT) COMPARED TO CONVENTIONAL RADIOTHERAPY?}

Although limb neoplasms are rare diseases, which makes it difficult to conduct prospective and randomized studies for a dosimetric comparison between radiotherapy techniques, there are some experimental or observational studies of better consistency showing that both IMRT and conformal radiotherapy are superior to radiotherapy as they allow the evaluation of irradiated treatment volumes and doses in normal structures of interest such as bones, surgical wound, graft flaps and skin bands, in both adults and children with indication of pre- or postoperative radiotherapy. Six studies have shown that the dose of radiation in areas of risk is greatly reduced when IMRT or conformal technique are used compared to conventional radiotherapy. ${ }^{2-7}(\mathbf{C})$

Three more recent studies have demonstrated superiority of the IMRT technique compared to conformal radiotherapy. All of them found greater conformity and dose homogeneity in treatment volumes and dose reduction for risk structures. ${ }^{8-10}(\mathbf{C})$

Robinson et al. compared the dosimetric plans of 11 patients with endometrial tumors between conventional radiotherapy and conformal radiotherapy. The volume of treatment with conformal radiotherapy was reduced in all patients. Normal muscle volume was reduced by $30 \%$ in thigh tumors. Likewise, the volumes of femur, tibia/fibula and ileum were reduced by $38 \%, 18 \%$ and $14 \%{ }^{2}$ (C) Stewart et al. updated these results by analyzing ten patients submitted to adjuvant radiotherapy after limb-preserving surgical resection due to thigh sarcomas, comparing conformal radiotherapy and IMRT. For all patients, the IMRT plans showed better conformity than those of conformal radiotherapy. IMRT allowed the release of a lower dose in the femur and normal tissue. In patients with lesions extending into the pelvis, bladder, rectum and bowel doses were also minimized. For the other parameters, there was no difference between techniques. ${ }^{6}(\mathbf{C})$

Sladowska et al. also presented the comparative results between IMRT and conformal radiotherapy in ten patients with thigh sarcomas. IMRT demonstrated better dose conformity in the target volume, especially when concave dose distribution was required. It also promoted a decrease 
of $31 \%$ in the maximum and average doses released in the femur, in all patients. ${ }^{10}(\mathbf{C})$

Due to the dosimetric data published in the literature, IMRT, when available, is the most recommended technique for the treatment of soft tissue tumors in the limbs, while conformal radiotherapy should be the least recommended technique.

\section{IS THERE LESS TOXICITY IN THE USE OF CONFORMAL RADIOTHERAPY OR IMRT COMPARED TO CONVENTIONAL RADIOTHERAPY FOR SOFT TISSUE TUMORS IN THE LIMBS?}

Alektiar et al. ${ }^{11}$ (B) analyzed the impact of IMRT on 41 adult patients with end-stage sarcomas treated at the Memorial Sloan-Kettering Cancer Center in New York between February 2002 and May 2005. The risk of complications was encouraging low, even including $25 \%$ of patients considered to be at high risk for the development of bone fractures after undergoing periosteal stripping or bone resection during surgery. Only two patients (4.8\%) developed grade 1 and 2 fractures and did not require surgical intervention for repair. Other complications such as edema and joint stiffness were also favorable when compared to conformal radiotherapy. Folker et al. ${ }^{12}(\mathbf{B})$, from the same institution, analyzed 319 patients with end-stage sarcomas treated with conservative limb surgery and adjuvant radiotherapy from 1996 to 2010 . Of these, 154 were treated with conformal radiotherapy and 165 with IMRT technique. Acute grade $\geq 2$ radiodermatitis was more frequent in patients treated with conformal radiotherapy ( $48.7 \%$ versus $31.5 \%$; $\mathrm{p}=0.002$ ). Grade $\geq 2$ chronic edema was also more frequent in patients who received conformal radiotherapy (14.9\% versus $7.9 \%$; $\mathrm{p}=0.05$ ). There was no difference in the incidence of pathological fracture, neuritis and joint stiffness between the two techniques. During the study period, no patient was treated with conventional radiotherapy.

O'Sullivan et al. ${ }^{13}$ (B) published the results of a phase 2 study involving 59 patients with end-stage sarcoma treated preoperatively with IMRT. The incidence of surgical wound complications was $30.5 \%$, less than the findings of the National Cancer Institute Canada study, which was $43 \%$ with conventional radiotherapy.

Clinical results with IMRT are still short-term and patients treated by different institutions require longer follow-up to consolidate results, but it can be inferred that these will not be inferior to those of conformal radiotherapy. The use of a conformal technique should constitute the minimum standard (least recommended) of radiotherapy for bone and soft tissue tumors of the limbs.

\section{IS THERE A DIFFERENCE IN EFFICACY BETWEEN CONFORMAL OR IMRT AND CONVENTIONAL RADIOTHERAPY?}

There are no randomized studies comparing the three planning and application techniques of radiotherapy. Due to the rarity of the disease and the multiplicity of possible presentations of limb tumors, it is unlikely that randomized trials for this evaluation will be performed.

In the American institution with the longest tradition in treating limb tumors (Memorial Sloan Kettering Cancer Center), from 1996 to 2010, 395 soft tissue sarcoma patients were treated with limb-preserving surgery and radiotherapy. ${ }^{12}$ (B) All patients after 2002 were treated with IMRT. Their study is the largest existing and compares the conformal and IMRT techniques. Conventional radiotherapy was not used in this period. In the study, the first 154 patients received adjuvant conformal radiotherapy and the following 165 received IMRT. Analysis of patient and tumor characteristics showed that the IMRT group had significantly more risk factors for recurrence (greater percentage of surgeries with positive or low margins and higher percentage of high-grade tumors). Patients treated with IMRT had fewer local recurrences ( $8 \%$ versus $15 \%, \mathrm{p}=0.05$ ). In a detailed multivariate analysis, IMRT remained a protective factor of independent recurrence (HR=0.458; CI 0.235-0.891).

A Canadian study compared a group of patients treated with IMRT in a phase 2 protocol with another group previously treated with conformal radiotherapy in a randomized study from the same institution. ${ }^{14}(\mathbf{B})$ The analysis showed 5-year recurrence-free survival of $88 \%$ in the IMRT-treated group and $89 \%$ of the historical control with conformal radiotherapy.

A study in children with rhabdomyosarcoma treated in a prospective protocol from the American Children's Oncology Group showed improvement in target volume coverage with IMRT. However, this did not translate into improvement of disease control results compared to conformal radiotherapy..$^{14}(\mathbf{C})$

There is therefore no evidence of good quality that demonstrates greater efficacy between IMRT and conforming techniques, making it impossible at this moment to recommend one over another. Regarding conventional radiotherapy, despite the absence of evidence of good quality, we recommend whenever possible that this technique not be used, due to its inability to allow evaluation of risk structures. In addition, there is a great risk of geographic error of the target to be irradiated due to the complex tissue irradiation needs in limb tumors. 


\section{Conclusion}

Bone and soft tissue tumors of the limbs are diseases with very variable presentation (histology, stage, volume of disease and location). Surgery is the treatment of choice and, because of the highly variable locations, it may not be feasible in many cases or it may be performed with minimally recommendable oncological principles.

The disease is a challenge for treatment and it is highly unlikely that randomized trials testing radiotherapy techniques will be performed anywhere in the world. There will probably never be high quality level 1 evidence for a decision on the radiotherapy technique and, thus, other criteria should be used for recommendations.

Conventional technique does not allow the physician to properly view the region being treated, nor the organs at risk. It does not allow international and national recommendations for doses in organs at risk because it is not possible to assess distribution. It is also subject to gross target location errors due to rotations of the limb that will be irradiated, which places the muscle bundle in an unconventional position. In any service that has the possibility of guiding the treatment using CT scans, we strongly recommend that the conventional technique be definitively abandoned as it has been for many years in developed countries.

If available, IMRT is superior to the others and potentially has a greater ability to control disease with lower toxicity. Due to a lack of clinical evidence, the minimally recommended radiotherapy technique to preserve the safety of patients with soft tissue tumors of the limbs is the conformal one.

\section{Conflict of interest}

The authors declare no conflict of interest.

\section{References}

1. Almeida CE, Haddad CK, Ferrigno R. A evolução técnica da radioterapia externa. In: Sociedade Brasileira de Radioterapia. Radioterapia Baseada em Evidências. Recomendações da Sociedade Brasileira de Radioterapia. 1. ed. São Paulo: SBRT; 2010. Chapter 2. p. 21-6.

2. Robinson MH, Bidmed AM, Harmer CL. Value of conformal planning in the radiotherapy of soft tissue sarcoma. Clin Oncol (R Coll Radiol).1992; 4(5):290-3.

3. Verhey LJ. Comparison of three-dimensional conformal radiation therapy and intensity-modulated radiation therapy systems. Semin Radiat Oncol. 1999; 9(1):78-98.

4. Patel S, DeLaney TF. Advanced-technology radiation therapy for bone sarcomas. Cancer Control. 2008; 15(1):21-37.

5. DeLaney TF, Trofimov AV, Engelsman M, Suit HD. Advanced-technology radiation therapy in the management of bone and soft tissue sarcomas. Cancer Control. 2005; 12(1):27-35.

6. Stewart AJ, Lee YK, Saran FH. Comparison of conventional radiotherapy and intensity-modulated radiotherapy for post-operative radiotherapy for primary extremity soft tissue sarcoma. Radiother Oncol. 2009; 93(1):125-30.

7. Hua C, Gray JM, Merchant TE, Kun LE, Krasin MJ. Treatment planning and delivery of external beam radiotherapy for pediatric sarcoma. Int J Radiat Oncol Biol Phys. 2008; 70(5):1598-606.

8. Chan MF, Chui CS, Schupak K, Amols H, Burman C, Ling CC. The treatment of large extraskeletal chondrosarcoma of the leg: comparison of IMRT and conformal radiotherapy techniques. J Appl Clin Med Phys. 2001; 2(1):3-8.

9. Griffin AM, Euler CI, Sharpe MB, Ferguson PC, Wunder JS, Bell RS, et al. Radiation planning comparison for superficial tissue avoidance in radiotherapy for soft tissue sarcoma of the lower extremity. Int J Radiat Oncol Biol Phys. 2007; 67(3):847-56.

10. Śladowska A, Hetnał M, Dymek P, Kabat D, Kisielewicz K, Wawrzak M, et al. Application of IMRT in adjuvant treatment of soft tissue sarcomas of the thigh - Preliminary results. Rep Pract Oncol Radiother. 2011; 16(3):110-4.

11. Alektiar KM, Brennan MF, Healey JH, Singer S. Impact of intensitymodutaded radiation therapy on local control in primary soft-tissue sarcoma of the extremity. J Clin Oncol. 2008; 26(20):2440-4.

12. Folkert MR, Singer S, Brennan MF, Kuk D, Qin LX, Kobayashi WK, et al. Comparison of local recurrence with conventional and intensity-modulated radiation therapy for primary soft-tissue sarcomas of the extremity. J Clin Oncol. 2014; 32(29):3236-42.

13. O'Sullivan B, Griffin AM, Dickie CI, Sharpe MB, Chung PW, Catton CN, et al. Phase 2 study of preoperative image-guided intensity-modulated radiation therapy to reduce wound and combined modality morbidities in lower extremity soft tissue sarcoma. Cancer. 2013; 119(10):1878-84.

14. Lin C, Donaldson SS, Meza JL, Anderson JR, Lyden ER, Brown CK, et al. Effect of radiotherapy techniques (IMRT vs. 3D-CRT) on outcome in patients with intermediate-risk rhabdomyosarcoma enrolled in COG D9803-A report from the Children's Oncology Group. Int J Radiat Oncol Biol Phys. 2012; 82(5):1764-70. 